\title{
Future Opportunities at the Facility for Rare Isotope Beams
}

\author{
Bradley M. Sherrill ${ }^{1, *}$ \\ ${ }^{1}$ Facility for Rare Isotope Beams, Michigan State University, East Lansing, MI 48824, USA
}

\begin{abstract}
This paper overviews the Facility for Rare Isotope Beams, FRIB, its construction status at the time of the conference, and its scientific program. FRIB is based on a high-power, heavy-ion, superconducting linear accelerator that is designed to deliver at least $400 \mathrm{~kW}$ at $200 \mathrm{MeV} / \mathrm{u}$ for all stable-ion beams and produce a large fraction of all possible isotopes of the elements. A three-stage fragment separator will separate rare isotope beams for use in experiments at high energy or stopped and reaccelerated to up to $10 \mathrm{MeV} / \mathrm{u}$. The facility is expected to have first beams in 2021. An overview of the planned scientific program, experimental capabilities, and equipment initiatives are presented.
\end{abstract}

\section{Introduction and Overview of the Facility for Rare Isotope Beams}

Creation and study of rare isotopes, isotopes of the elements that are not normally found on Earth, is a key element of the goal to accurately model the atomic nucleus. Rare isotopes provide insights into nuclear structure, benchmark models, and can be used to determine model parameters. Accurate models of nuclear properties and the reactions of atomic nuclei, with sufficient precision, would be revolutionary in our ability to understand the universe and in our ability to apply nuclei to solve societal problems. It would remove nuclear physics uncertainties in the application of nuclei to probe for the fundamental interactions of nature.

One of the major new facilities to make beams of rare isotopes is the Facility for Rare Isotope Beams, FRIB [1]. Establishment of FRIB as a user facility open to all researchers is supported by the Department of Energy Office of Science. FRIB is based on a high-power, heavy-ion, superconducting linear accelerator that is designed to deliver at least $400 \mathrm{~kW}$ at $200 \mathrm{MeV} / \mathrm{u}$ for all stable-ion beams. For reference, $400 \mathrm{~kW}$ of a ${ }^{238} \mathrm{U}$ beam corresponds to $8 \mathrm{p} \mu \mathrm{A}$ or $5 \times 10^{13}$ ions/s. A three-stage fragment separator [2] will separate rare isotope beams in-flight. Beams can be used in experiments at high energy or stopped and reaccelerated to up to $10 \mathrm{MeV} / \mathrm{u}$. The availability of rare isotope beams at energies from tens of $\mathrm{kV}$ to 200 $\mathrm{MeV} / \mathrm{u}$ is one of the key features of FRIB. Particularly unique, is the plan to reaccelerate beams after in-flight separation and stopping.

An overview of the facility is shown in Figure 1. The callout circles illustrate various parts of the facility. Ions are created in a pair of ECR ion sources and are accelerated in a

\footnotetext{
* Corresponding author: sherrill@frib.msu.edu
} 
superconducting linear accelerator that is folded two times. After the first section the ions are stripped in a liquid lithium charge stripper. Rare isotopes are produced in a rotating graphite target and the unused beam is stopped in a rotating drum of water. An interesting possibility is to harvest otherwise unused isotopes from the water drum [3]. Isotopes are separated, inflight with a fragment separator [2] and delivered to experimental areas for fast, stopped, and reaccelerated beams. Beams are stopped in pure helium and reaccelerated in the ReA3/6 reaccelerators [4]. The reaccelerators were developed at the National Superconducting Cyclotron Laboratory supported by the USA National Science Foundation [5].

FRIB is expected to have first beams in 2021. Once operational the facility is projected to take five years to increase the beam power from $10 \mathrm{~kW}$ in the first year to $400 \mathrm{~kW}$ in year five. FRIB is planned to have ${ }^{238} \mathrm{U},{ }^{48} \mathrm{Ca},{ }^{78} \mathrm{Kr},{ }^{82} \mathrm{Se},{ }^{124} \mathrm{Xe},{ }^{18} \mathrm{O},{ }^{86} \mathrm{Kr},{ }^{16} \mathrm{O}$, and ${ }^{36} \mathrm{Ar}$ primary beams in the first year. Four to five new primary beams will be added each year.

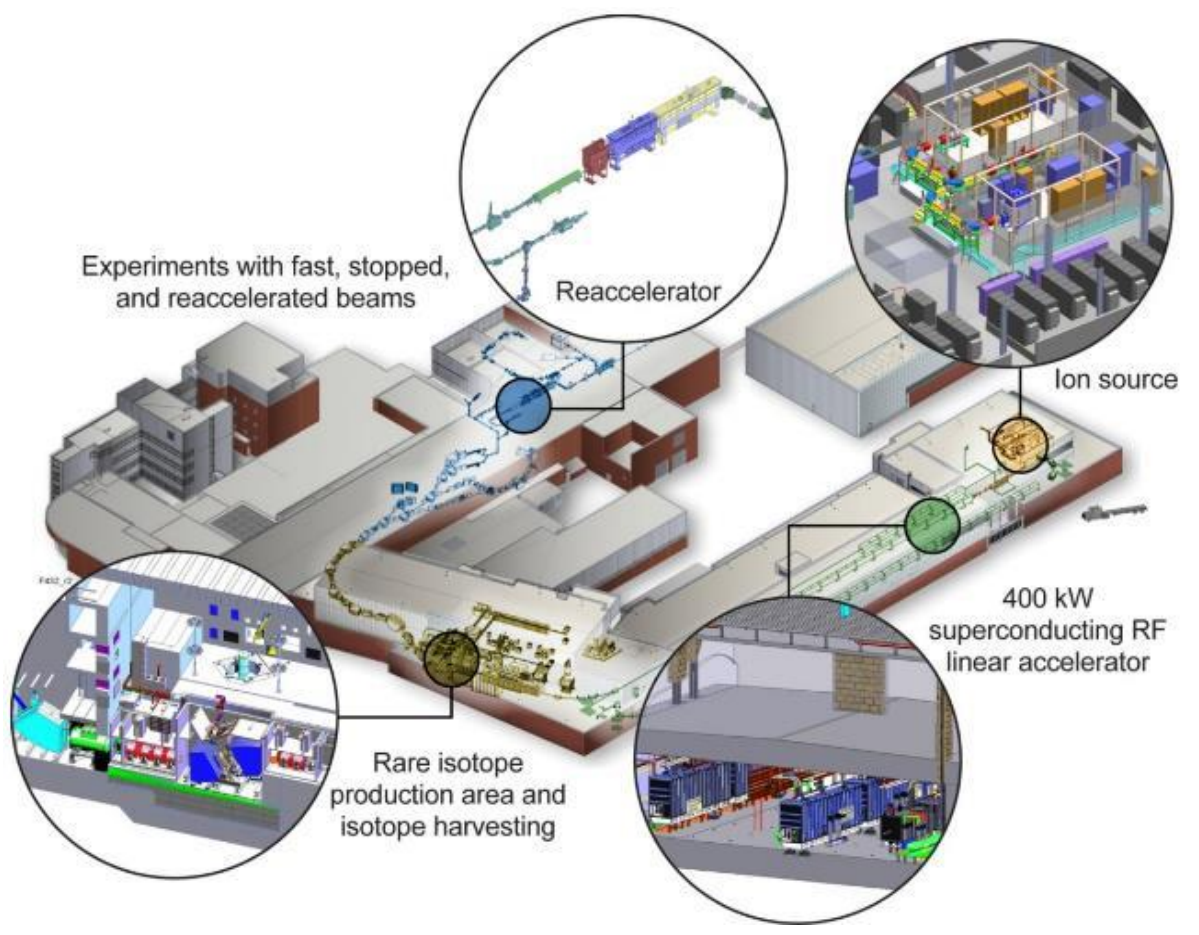

Fig. 1. Overview of the FRIB facility. Stable ions are created in the ECR ions sources at the right and accelerated in the FRIB superconducting LINAC up to $200 \mathrm{MeV} / \mathrm{u}$ for uranium ions with beam power up to $400 \mathrm{~kW}$. The beam strikes a production target and the rare isotopes produced are separated inflight and delivered to an experimental area with capabilities for study of fast, stopped, and reaccelerated beams. Reaccelerated beams will be available at FRIB at up to $10 \mathrm{MeV} / \mathrm{u}$ based on the current ReA6 facility.

\section{Scientific Program}

The scientific program at FRIB will have four main areas of study; nuclear structure, exploration of the role of nuclei in the cosmos, use of atomic nuclei to provide tests of nature's fundamental symmetries, and the applications of atomic nuclei to address societal issues [6]. FRIB will offer researchers the possibility to achieve the overarching goal to accurately model atomic nuclei, mentioned above. Furthermore, FRIB will make possible the measurement of a majority of key nuclear reactions needed for a quantitative understanding 
of the nuclear processes leading to the chemical makeup of the universe [7]. FRIB will enable tests of Nature's fundamental symmetries by providing the largest quantifies of key isotopes to measure nuclear EDMs, search for interactions beyond $\mathrm{V}-\mathrm{A}$, or measure annapole moments [8].

The scientific potential of FRIB is made possible by the vast range of isotope beams that the facility will produce. The intensity, displayed as the LOG of the rate per second, of the projected beams is illustrated in Figure 2. The useful range of intensities varies from $10^{12} / \mathrm{s}$ near the stable beam isotopes to production rates of atoms/week. The rates are calculated with the program LISE++ using the EPAX2.15 cross section systematics for fragmentation and the LISE-FIS model for fission [9]. For reference the dark line shows the approximate region of known nuclei. FRIB should be able to reach the dripline for elements up to approximately 30 and many of the isotopes relevant for the r-process. The high reaccelerated rates of proton rich nuclei up to mass 100 will allow many of the key reactions important for modelling X-ray bursts to be measured directly using SECAR [7].

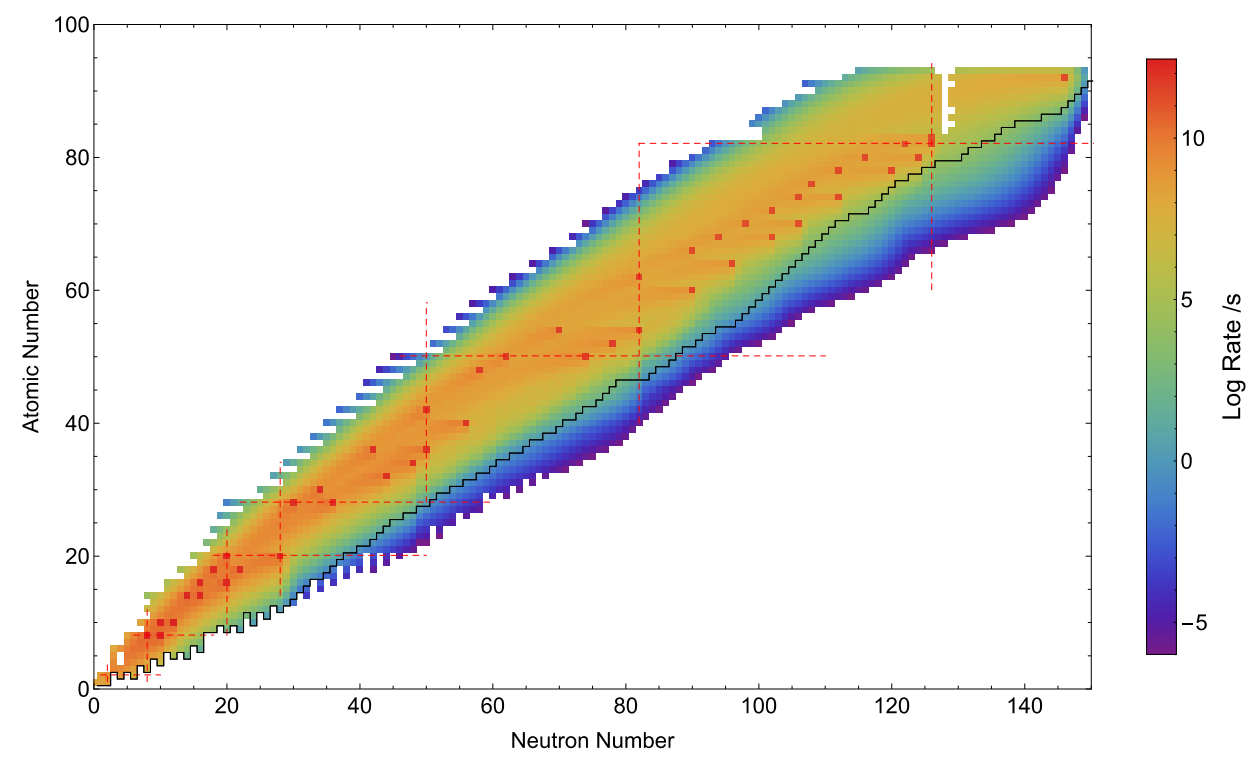

Fig. 2. Projected rare isotope beam intensities for fast beams that will be available at FRIB. Rates for stopped and reaccelerated beams will be reduced by the stopping and reacceleration efficiencies. The rates have been calculated by the program LISE++ with the assumptions described in the text. The rates for very neutron-rich nuclei are uncertain.

During the production of an isotope for a particular experiment, there will be the opportunity to collect unused isotopes by harvesting them from the beam dump or at various locations in the fragment separator. For special applications, such as collection of a sample for neutron irradiation where isotopic purity is important it is possible to have dedicated runs for collection. These options would allow micro-mole samples of certain isotopes to be collected and used at other facilities, such as neutron-irradiation facilities to study neutroncapture reactions. The harvested isotopes will also likely be of interest for a wide variety of applications [3].

\section{Experimental Equipment}

A wide variety of experimental equipment has been developed or is being proposed by the user community. These initiatives are described at the FRIB User Organization, FRIBUO 
web site [10]. The FRIBUO has approximately 1,400 members, with 250 institutions and 51 countries represented as of September 2017. A summary of the major experimental equipment items is listed in Table 1. A schematic layout of the FRIB equipment areas is shown in Figure 3. FRIB builds on the experiment that has been developed at the National Superconducting Cyclotron Laboratory, which is supported by the USA National Science Foundation.

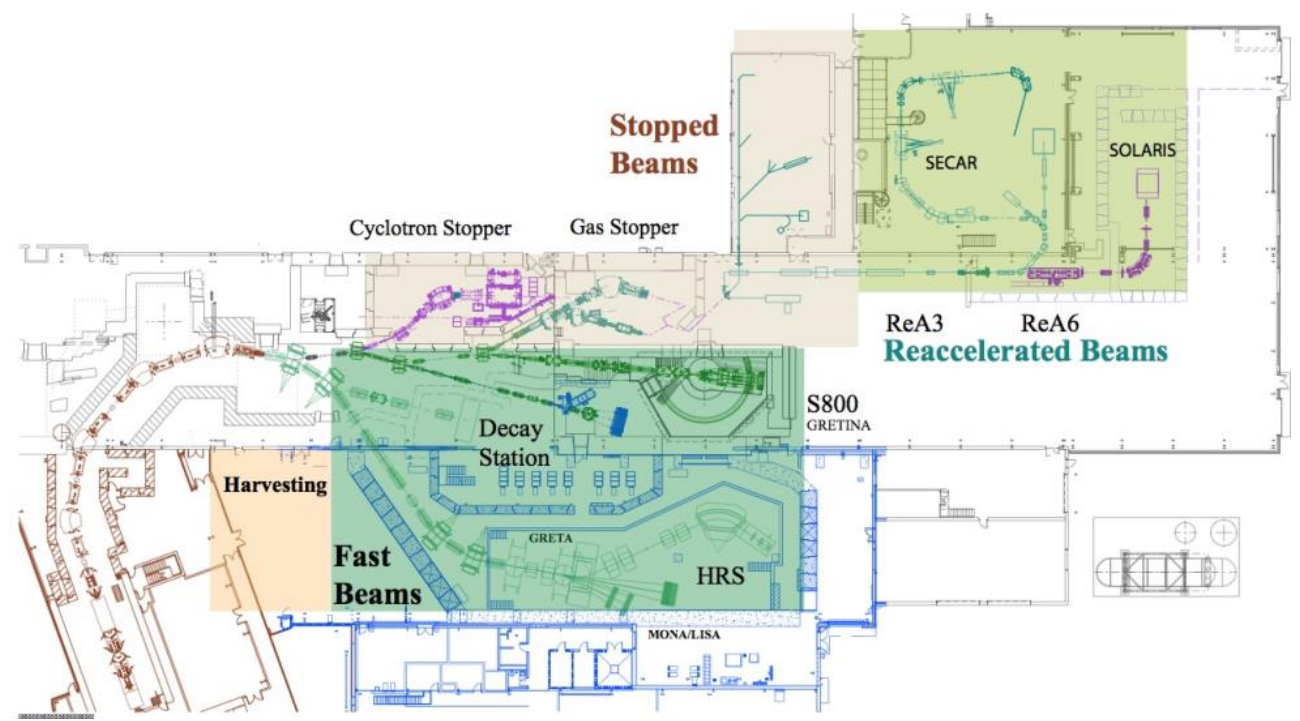

Fig. 3. Schematic layout of the FRIB facility showing the various experimental equipment planned.

Table 1. List of key experimental equipment planned for FRIB.

\begin{tabular}{|c|c|c|c|}
\hline $\begin{array}{l}\text { Equipment } \\
\text { Item }\end{array}$ & Description and Program & Status & Ref \\
\hline HRS & $\begin{array}{l}\text { Bend reaction products with rigidities up to } 8 \\
\text { Tm, which allows efficient use of FRIB beams } \\
\text { and luminosity gains of 20x }\end{array}$ & $\begin{array}{l}\text { Conceptual Design } \\
\text { Report in } 2018\end{array}$ & [11] \\
\hline $\begin{array}{l}\text { Beta Decay } \\
\text { Station }\end{array}$ & $\begin{array}{l}\text { Study of radioactive decay of rare isotopes } \\
\text { provide a sensitive test of nuclear structure } \\
\text { and key for r-process modelling }\end{array}$ & White Paper in 2018 & [12] \\
\hline GRETA & $\begin{array}{l}\text { Germanium-based gamma-ray array with 4pi } \\
\text { coverage and position sensitivity to allow } \\
\text { Doppler reconstruction }\end{array}$ & $\begin{array}{l}\text { Funded, completion } \\
\quad \text { in } 2025\end{array}$ & [13] \\
\hline SECAR & $\begin{array}{l}\text { Recoil mass separator to allow direct } \\
\text { measurement of key nuclear reaction rates }\end{array}$ & $\begin{array}{l}\text { Funded, completion } \\
\text { in } 2020\end{array}$ & [14] \\
\hline SOLARIS & $\begin{array}{l}\text { Solenoid spectrometer for measurement of } \\
\text { nuclear reactions with reaccelerated beams }\end{array}$ & White Paper in 2018 & [15] \\
\hline ISLA & $\begin{array}{l}\text { Time-of-flight mass separator for tagging of } \\
\text { nuclear reactions with reaccelerated beams }\end{array}$ & White Paper in 2015 & [16] \\
\hline $\begin{array}{l}\text { Existing } \\
\text { Equipment }\end{array}$ & $\begin{array}{l}\text { Many pieces of experimental equipment } \\
\text { already exist and will be used at FRIB for day- } \\
\text { one experiments. Examples are the S800, } \\
\text { GRETINA, LEBIT, and BECOLA }\end{array}$ & Existing & [17] \\
\hline
\end{tabular}




\section{Summary}

FRIB will provide a vast array of rare isotopes that will allow scientists to create a comprehensive model of nuclei, determine nuclear reactions and properties key to modelling the universe, provide rare isotopes to test fundamental symmetries, and provide a fast path to many isotopes that can be important for developing new techniques in other fields. The reach of FRIB will be enabled by the $400 \mathrm{~kW}$ heavy ion, superconducting driver LINAC. It is anticipated that first experiments will begin by 2022. A wide array of experimental equipment is under development by the FRIB user community. Existing equipment will be ready for day-one experiments.

Acknowledgements: Establishment of the Facility for Rare Isotope Beams is supported by the Department of Energy Office of Science. The project is led by the FRIB Laboratory Director Prof. Thomas Glasmacher and the Project Manager Prof. Paul Mantica. The work described here is carried out by the FRIB project team and not the author. Plans for the scientific program described here has been developed by the FRIB user community represented by the FRIB User Organization, FRIBUO.

\section{References}

1. J. Wei et al., LINAC12: Proceedings of the 26th International Linear Accelerator Conference (2012); http://accelconf.web.cern.ch/accelconf/linac2012/index.htm

2. M. Hausmann, A. M. Aaron, A. M. Amthor, M. Avilov, L. Bandura, R. Bennett, G. Bollen, T. Borden, T. W. Burgess, S. S. Chouhan, V. B. Graves, W. Mittig, D. J. Morrissey, F. Pellemoine, M. Portillo, R. M. Ronningen, M. Schein, B. M. Sherrill, A. Zeller, Nucl. Instr. and Methods Section B 317(PART B) 349 (2013)

3. T. Mastren, A. Pen, G.F. Peaslee, N. Wozniak, S. Loveless, S. Essenmacher, L.G. Sobotka, D. J. Morrissey, S.E. Lapi, Nature Scientific Reports 46706 (2014)

4. D. Leitner, Nucl. Instr. and Methods Section B 317 (PART B) 235 (2013)

5. A. Gade and B. M. Sherrill, Physica Scripta 91033006 (2016)

6. A.B. Balantekin, J. Carlson, D.J. Dean, G.M. Fuller, R.J. Furnstahl, M. Hjorth-Jensen, R.V.F. Janssens, B.-A. Li, W. Nazarewicz, F.M. Nunes, W.E. Ormand, S. Reddy, B.M. Sherrill, Mod. Phys. Lett. A 2911 (2014)

7. J. Carlson et al., Prog. Part. Nucl. Physics 9468 (2017)

8. Fundamental Symmetry Tests with Nucleons, Nuclei, and Atoms: A Snowmass Report, K. Kumar et al., arXiv:1312.5416v1 [hep-ph] 19 Dec 2013

9. https://groups.nscl.msu.edu/frib/rates/fribrates.html

10. http://fribusers.org/

11. http://hrs.lbl.gov/science

12. https://groups.nscl.msu.edu/fribdecaystation/

13. http://greta.lbl.gov/documents/GRETA-WhitePaper.pdf

14. http://fribastro.org/SECAR/REFERENCE/SECAR-design.pdf

15. https://www.anl.gov/phy/group/solaris

16. http://www.lecmeeting.org/whitepapers/White paper Isla recoil complete.pdf

17. http://fribusers.org/documents/2014/FRIB_EQUIPMENT_whitepaper.pdf 